\title{
Sensory Loss in Hospital-Admitted People with Stroke: Characteristics, Associated Factors, and Relationship with Function
}

\author{
Sarah F. Tyson, Marie Hanley, Jay Chillala, Andrea B. Selley, and Raymond C. Tallis
}

\begin{abstract}
Objective: To characterize the nature of sensory impairments after stroke, identify associated factors, and assess the relationships between sensory impairment, disability, and recovery. Methods: Prospective cross-sectional survey of 102 people with hemiparesis following their first stroke. Tactile and proprioceptive sensation in the affected arm and leg were measured using the Rivermead Assessment of Somatosensory Perception 2-4 weeks post-stroke. Demographics, stroke pathology, weakness, neglect, disability, and recovery were documented. Results: Tactile impairment was more common than proprioceptive $(p<$ .000 ), impairment of discrimination was more common than detection $(p<.000)$, and tactile sensation was more severely impaired in the leg than the arm $(p<.000)$. No difference in proprioception between the arm and leg $(p=.703)$ or between proximal and distal joints $(p=.589, p=.705)$ was found. The degree of weakness and the degree of stroke severity were significantly associated with sensory impairment; demographics, stroke side and type, and neglect were not associated. All the sensory modalities were significantly related to independence, mobility, and recovery $(r=0.287[p<.011]$ to $r=0.533[p<$ $.000])$. Conclusion: Sensory impairments of all modalities are common after stroke, although tactile impairment is more frequent than proprioceptive loss, especially in the leg. They are associated with the degree of weakness and the degree of stroke severity but not demographics, stroke pathology, or neglect, and they are related to mobility, independence in activities of daily living (ADL), and recovery.
\end{abstract}

Key Words: Sensation-Proprioception-Stroke outcomes

S ensory loss is thought to be common after a stroke, ${ }^{1}$ and the restoration of impaired sensation is an important aspect of stroke rehabilitation. It is considered a precursor to the recovery of movement and functional activity, and it is an important part of widely used physiotherapy approaches such as Bobath ${ }^{2,3}$ (known as Neurodevelopment Therapy in the United States) and

From the Centre for Rehabilitation and Human Performance Research, University of Salford, United Kingdom (SFT) and the University of Manchester, United Kingdom (MH, JC, ABS, RCT).

Address correspondence to Sarah Tyson, Centre for Rehabilitation and Human Performance Research, University of Salford, Mary Seacole Building, Frederick Rd Campus, Salford M6, UK. E-mail: s.tyson@ salford.ac.uk.

DOI: $10.1177 / 1545968307305523$
Brunnstrom. $^{3}$ There is little objective data surrounding sensory impairment after stroke. There are no studies in the published literature that adequately describe the nature of sensory deficits. Previous studies have described selected study populations such as people who can walk or stand unaided ${ }^{4-6}$ or have measured only one limb ${ }^{5-7}$ or only one sensory modality. ${ }^{5,-11}$ Others describe the relationship between sensory impairments and function and/or recovery. ${ }^{4-12}$ Tactile sensation and proprioception are the most common modalities tested, but most studies used measurement tools in which the psychometric properties have not been fully demonstrated. . $^{4-12}$

We undertook a study to describe the nature of sensory impairments after stroke. We characterized the nature of sensory impairments after stroke, identified the factors associated with sensory impairment, and assessed the relationships between sensory impairment, disability, and recovery. Our research questions addressed the differences in the frequency of proprioceptive and tactile deficits and the relationships between proprioceptive and tactile impairments. We assessed whether sensory impairments were more severe in the arm than leg, and whether sensory impairments were more severe distally than proximally. We identified factors that influence the severity of sensory impairments and examined the relationship between sensory impairments, function, and recovery. This was part of a larger study to investigate the relationships between impairments, disabilities, and recovery after stroke, with particular reference to balance disability and mobility function. ${ }^{13-15}$

\section{METHODS}

\section{Design and Participants}

We undertook a prospective cross-sectional survey of consecutive stroke patients admitted to National Health Service Trusts in Greater Manchester (UK). We included patients with weakness following a first-time anterior circulation stroke. Patients who had had a previous stroke; could not give informed consent; were too ill to participate; had another neurological condition that could affect 
balance or mobility; or had another condition that severely limited their balance or mobility prior to the stroke were excluded.

One hundred two participants were recruited. Only 9 patients who met the inclusion criteria declined to participate. There were 54 men. Mean age was 70.7 (SD 12.6) years. Sixty-five subjects had a left-sided weakness. Eighty-nine subjects had an ischemic stroke, 26 had a total anterior circulation stroke (TACS), 40 had partial anterior circulation stroke (PACS), and 36 had a lacunar anterior circulation stroke (LACS). Their median score on the Barthel Index was 11 (IQR 6-16), and their median score on the Rivermead Mobility Index was 4 (IQR 1-8). Follow-up assessment of the Barthel Index and Rivermead Mobility Index was obtained from 75 participants at 3 months post-stroke; $45(60 \%)$ were men; $48(65 \%)$ had a left hemiplegia; and $16(22 \%)$ had suffered a TACS, $27(37 \%)$ a PACS, and $42(42 \%)$ a LACS. Their mean age was 71 years (SD 12.2), their median Barthel Index score was 16 (IQR 7.5-18), and their median Rivermead Mobility Index score was 8 (IQR 4-12). Of the nonresponders, 2 (2\%) had died, 4 $(4 \%)$ were being treated primarily for another illness, 1 had suffered another stroke, 9 (9\%) declined to participate, and $13(13 \%)$ were untraceable because they were no longer living at the same address.

\section{Measurement}

After informed consent had been obtained, participants were tested 2-4 weeks after their stroke in a single assessment session at the hospital bedside or physiotherapy treatment area. Data were collected by 1 of 4 assessors, 2 senior neurological physiotherapists (ST, AS) and 2 geriatricians $(\mathrm{MH}, \mathrm{JC})$. Interrater reliability was ensured through joint training sessions and regular review sessions when the scoring methods were reviewed and discussed, and any differences in scoring methods resolved. At 3 months post-stroke, a follow-up assessment of the recovery of function (independence in ADL and mobility) was made using the modified Barthel Index and Rivermead Mobility Index administered by postal questionnaire. The Barthel Index and Rivermead Mobility Index were posted to participants with a stamped, addressed envelope for the replies. Nonresponders were contacted by telephone, and those who wished to continue with the study were sent another copy of the form or, when the participant preferred, the form was completed over the telephone.

At the main testing session, the data collected were:

demographic details: age, sex, prestroke disability (Rankin Scale $^{16}$ );

stroke pathology: stroke type (ischemic or hemorrhagic from CT scan), location (left or right from CT scan), and severity (Oxford Community Stroke Project classification ${ }^{17}$ );

neurological impairments: neglect (star cancellation and line bisection tests ${ }^{18}$ ); weakness (Motricity Index ${ }^{19}$ ) and sensation (Rivermead Assessment of Somatosensory Performance $\left.{ }^{20}\right)$; and

Function-independence in ADL (Barthel Index ${ }^{21}$ ) and functional mobility (Rivermead Mobility Index ${ }^{22}$ ).

Sensation was tested using parts of the Rivermead Assessment of Somatosensory Perception (RASP), which is a measurement tool designed to operationalize the clinical assessment of sensation in people with neurological conditions. ${ }^{20}$ Good interrater and test-retest reliability and face, content, discriminant, and concurrent validity have been described for the modalities used in this study. ${ }^{20}$ Two modalities (proprioception and touch) and 2 aspects of these modalities (detection and discrimination) were measured on the affected side. For proprioception, the affected elbow, wrist, thumb, ankle, and big toe were tested through full range. For "detection of proprioception," the participants were asked to indicate when they felt the joint moving, and for "discrimination of proprioception" they were asked to indicate the direction of movement. This was performed with the participants' eyes closed during passive flexion and extension of each joint. For touch, the foot (sole and dorsal aspects) and hand (palmar and dorsal aspects) of the affected side were tested with the participants' eyes closed. For "detection of touch," participants indicated when they could detect the tester lightly touching their hand or foot. For "discrimination of touch," the participant indicated the location of the touch. To standardize the pressure applied when touching the participant, the "Neurometer" from the RASP was used. This device looks very much like a ball-point pen with a retractable nib. The "nib" is spring loaded. It is applied to the skin and pressure applied to a predefined point, thereby applying a standardized pressure. For each of the sensory modalities, the tests were repeated 6 times, with the participant saying when or where they could feel the touch or movement. The number of times the participant correctly identified the sensation was counted. On the basis of the number of correct "scores," patients were arbitrarily categorized as follows: $0-1=$ absent; $2-5=$ impaired; $6=$ intact. The total scores for each limb (proprioception plus touch) and each modality (proprioceptive detection, proprioceptive discrimination, tactile detection, and tactile discrimination) were calculated and categorized using the same method.

\section{Analysis}

Descriptive statistics were used to describe the sensory impairments. Nonparametric statistics (Wilcoxon 
Table 1. The Frequency and Severity of Tactile Sensation and Proprioceptive Impairments after Stroke

\begin{tabular}{lcccc}
\hline & Intact & Impaired & Absent & $p$ Value \\
\hline Tactile sensation & 35 & 41 & 26 & .000 \\
Proprioception & 74 & 17 & 11 & \\
$\begin{array}{l}\text { Detection (tactile + } \\
\quad \text { proprioception) }\end{array}$ & 54 & 34 & 14 & .000 \\
$\quad$ Discrimination (tactile + & 36 & 45 & 21 & \\
$\quad$ proprioception) & & & & \\
$\begin{array}{l}\text { Proprioceptive detection } \\
\text { Proprioceptive }\end{array}$ & 80 & 12 & 10 & .004 \\
$\quad$ discrimination & 74 & 15 & 13 & \\
$\quad$ Tactile detection & 56 & 21 & 25 & .000 \\
Tactile discrimination & 36 & 24 & 42 & \\
\hline
\end{tabular}

and Friedman tests) compared the different groups. Crosstabulation assessed the relationship between proprioception and tactile sensation modalities. Linear regression analysis using the enter method assessed the factors influencing sensory impairment. Sensory impairment was the dependent variable, and demographic factors, stroke pathology factors, and other stroke impairments were independent factors. Spearmann's rho was used to assess the relationship between sensory modalities (overall sensation, proprioception, and tactile sensation), function (independence in ADL and mobility), and recovery (of independence in ADL and mobility at 3 months post-stroke).

\section{RESULTS}

Sixty-seven participants had impaired or absent tactile sensation, whereas 28 participants had impaired or absent proprioception. These differences were highly significant $(p<.000)$. Discrimination was more impaired than detection $(p<.000)$ for both proprioception $(p<$ $.003)$ and tactile sensation $(p<.000)$ (Table 1$)$. There was a close association between the detection modalities and the discrimination modalities, with most participants obtaining the same score for both modalities (Table 2).

There was no difference between arm and leg proprioception $(p<.703)$, or proprioceptive detection and discrimination $(p<.963$ and $p<.581$, respectively). However, the leg was significantly more impaired than the arm on all tactile sensation modalities: overall tactile sensation $(p<.016)$, tactile detection and tactile discrimination $(p<.038$ and $p<.026$, respectively), and in overall total sensation (proprioception plus tactile sensation) $(p<.046)$ (Table 3$)$.

Upper limb proprioception (detection and discrimination) was measured at the elbow, wrist, and thumb. Results from the Friedman's test showed that there were
Table 2. Cross-tabulation between Proprioceptive and Tactile Modalities

\begin{tabular}{lcccc}
\hline & \multicolumn{4}{c}{ Proprioceptive Detection } \\
\cline { 2 - 5 } & Intact & Impaired & Absent & Total \\
\hline Tactile detection & & & & \\
$\quad$ Intact & 54 & 2 & 0 & 56 \\
Impaired & 25 & 11 & 1 & 37 \\
Absent & 0 & 0 & 9 & 9 \\
Total & 79 & 13 & 10 & 102 \\
\hline & \multicolumn{2}{c}{ Proprioceptive Discrimination } \\
\cline { 2 - 5 } & Intact & Impaired & Absent & Total \\
\hline Tactile discrimination & \multicolumn{4}{c}{} \\
Intact & 36 & 0 & 0 & 36 \\
Impaired & 29 & 6 & 0 & 35 \\
Absent & 9 & 11 & 11 & 31 \\
Total & 74 & 17 & 11 & 102 \\
\hline
\end{tabular}

Table 3. Comparison of Proprioception and Tactile Sensation in the Upper and Lower Limb after Stroke

\begin{tabular}{lrrrr}
\hline & Intact & Impaired & Absent & $p$ Value \\
\hline Total (arm) & 44 & 42 & 16 & .046 \\
Total (leg) & 38 & 45 & 19 & \\
Proprioception (leg) & 77 & 14 & 11 & .703 \\
Proprioception (arm) & 80 & 9 & 13 & \\
Tactile (leg) & 38 & 36 & 28 & .016 \\
Tactile (arm) & 45 & 33 & 24 & \\
Proprioceptive & 83 & 8 & 11 & .963 \\
$\quad$ detection (leg) & & & & \\
Proprioceptive & 85 & 4 & 13 & \\
$\quad$ detection (arm) & & & & \\
Proprioceptive & 77 & 11 & 14 & .581 \\
$\quad$ discrimination (leg) & & & & \\
Proprioceptive & 80 & 8 & 14 & \\
$\quad$ discrimination (arm) & & & & \\
Tactile detection (leg) & 60 & 14 & 28 & .038 \\
Tactile detection (arm) & 69 & 9 & 24 & \\
Tactile discrimination (leg) & 39 & 18 & 45 & .026 \\
Tactile discrimination (arm) & 46 & 20 & 36 & \\
\hline
\end{tabular}

no significant differences between the joints for either detection $(p<.589)$ or discrimination $(p<.705)$ (Table 4$)$.

Three regression models were analyzed with overall sensation, proprioception, and tactile sensation as the dependent variables. For total sensation and tactile sensation, weakness and stroke severity were significant independent factors influencing sensory impairment. For proporioception, weakness was the only significant factor. Age, sex, premorbid disability, side and type of stroke, and neglect were not significant for any of the models (Table 5). 
Table 4. Showing the Proprioceptive Impairments in the Affected Elbow, Wrist, and Thumb

\begin{tabular}{lcccc}
\hline & Intact & Impaired & Absent & $p$ Value \\
\hline Detection & & & & .589 \\
Elbow & 87 & 0 & 15 & \\
Wrist & 88 & 0 & 14 & \\
Thumb & 85 & 2 & 15 & \\
Discrimination & & & & .705 \\
Elbow & 80 & 6 & 15 & \\
Wrist & 81 & 5 & 15 & \\
Thumb & 80 & 6 & 15 & \\
\hline
\end{tabular}

There were moderate but significant relationships between the sensory modalities and mobility and independence in ADL in the acute stages $(r=0.416,[p<$ $.000]$ to $r=0.515[p<.000])$, and low-to-moderate significant relationship at follow-up 3 months after stroke $(r=0.287[p<.011]$ to $r=0.533[p<.000])$ (Table 6). In all cases, overall sensation showed a stronger correlation than proprioception or tactile sensation alone.

\section{DISCUSSION}

The results of this study show that sensory impairments are common after stroke. Tactile impairment is more frequent than impairment of proprioception; impairment of discrimination is more frequent than impairment of detection, and tactile sensation is more severely impaired in the leg than the arm. There is no difference in proprioception between the arm and leg and no difference in any sensory modality between proximal and distal joints. The degree of weakness and degree of stroke severity were factors significantly influencing sensory impairment; demographics, stroke pathology, and neglect were not significant.

This is the first paper to describe systematically the nature of sensory impairments after stroke. The frequency of sensory impairment is similar to previous estimates. ${ }^{1,12,23}$ In the present study, discrimination was found to be more frequently impaired than detection. This finding has been reported previously ${ }^{1}$ and is not surprising, as the discrimination of a stimulus is obviously more challenging than detection, since one needs to be able to detect a stimulus to discriminate its location or direction. Most participants obtained the same score for detection and discrimination modalities. This finding has been previously reported for discrimination modalities, ${ }^{1}$ but not for detection modalities.

The results of the present study demonstrate for the first time that tactile sensation is more impaired than proprioception in anterior circulation stroke patients. One explanation may be that the central nervous system (CNS) pathways associated with proprioception are

Table 5. Showing the Linear Regression Models for the Factors Influencing Sensory Impairments after Stroke

\begin{tabular}{|c|c|c|c|c|c|c|}
\hline & \multicolumn{2}{|c|}{$\begin{array}{l}\text { Overall Sensation } \\
\qquad \mathrm{R} 2=39 \%\end{array}$} & \multicolumn{2}{|c|}{$\begin{array}{l}\text { Proprioception } \\
\text { R2 }=26 \%\end{array}$} & \multicolumn{2}{|c|}{$\begin{array}{l}\text { Tactile Sensation } \\
\qquad \mathrm{R} 2=41 \%\end{array}$} \\
\hline & Beta Coefficient & $p$ Value & Beta Coefficient & $p$ value & Beta Coefficient & $p$ Value \\
\hline Age & -.030 & .729 & .038 & 690 & .016 & .849 \\
\hline Sex & .103 & .239 & -.123 & .209 & -.099 & .254 \\
\hline Pre-morbid disability & -.049 & .565 & .008 & .933 & .077 & .359 \\
\hline Side of hemiplegia & .019 & .818 & -.016 & .866 & .017 & .841 \\
\hline Infarct or haemorrhage & -.017 & .840 & .020 & .828 & .006 & .942 \\
\hline Stroke severity & .325 & .002 & -.195 & .084 & -.427 & .000 \\
\hline Weakness & .408 & .000 & -.366 & .001 & -.329 & .001 \\
\hline Neglect & .052 & .599 & -.049 & 655 & -.069 & .477 \\
\hline
\end{tabular}

Table 6. Spearman's Rho Correlation between Sensation, Proprioception, and Tactile Sensation, Independence in the Activities of Daily Living, Functional Mobility, and Recovery of Independence and Mobility

\begin{tabular}{lccr}
\hline & Sensation & Proprioception & Tactile Sensation \\
\hline Functional mobility & $0.515(p<.000)$ & $0.416(p<.000)$ & $0.500(p<.000)$ \\
Independence in ADL & $0.541(p<.000)$ & $0.456(p<.000)$ & $0.518(p<.001)$ \\
Recovery of functional mobility & $0.328(p<.003)$ & $0.317(p<.005)$ & $0.287(p<.011)$ \\
Recovery of independence in ADL & $0.533(p<.000)$ & $0.496(p<.000)$ & $0.495(p<.000)$ \\
\hline
\end{tabular}


more diffuse than those for tactile sensation or that they have more redundancy. Alternatively, the proprioceptive test may simply be easier than tests of tactile sensation.

Textbooks have stated that the arm is usually more severely impaired than the leg. ${ }^{24,25}$ The findings of the present study do not support this belief; we found no difference in proprioception between the limbs. Tactile sensation was more impaired in the lower limb than the upper. This difference was sufficient to indicate an overall difference in sensation between the upper and lower limbs. The lower limb showed more impairment. This result is surprising. The cortical sensory representation of the upper limb is much bigger than the lower limb, so one might expect it to be more vulnerable to impairment. The finding that the lower limb is more impaired than the upper limb may indicate that greater cortical representation implies greater redundancy, or that CNS pathways are more diffuse, or that they include greater bilateral projection. Alternatively, since the upper limb has a lower detection threshold than the lower limb, a much larger sensory impairment would be required in the upper limb than in the lower limb to cause a detection error.

The results of this study indicate that weakness and severity of stroke were significantly associated with sensory impairment; demographics, stroke pathology, and neglect were not significant. It was expected that stroke severity would be associated with sensory impairment, but not necessarily between weakness and sensation. Further research is necessary to investigate the nature of the relationship between motor and sensory impairment and the impact of the size and location of the lesion.

We found, as in other correlation studies and studies comparing different groups, ${ }^{4-10,26}$ that tactile sensation, proprioception, and overall sensation are related to mobility, independence in $\mathrm{ADL}$, and recovery, although the relationships were only weak-to-moderate. Numerous previous studies have shown that persons with stroke who have sensory impairments and motor impairments have a worse outcome than persons with motor impairments alone, ${ }^{9,11,27-31}$ and that persons with normal sensory function have a more prompt and robust recovery than persons with impaired sensation, ${ }^{9}$ to the extent that sensory impairment is included in prognostic scales to predict recovery from stroke. ${ }^{29,30}$ However, there is evidence to question whether sensory impairment is a cause or a cofactor in disability and recovery. Several studies using multiple regression analyses have found that sensory loss is a significant factor in different activities (gait, ${ }^{5}$ upper limb recovery, ${ }^{7}$ balance, ${ }^{14}$ mobility disability,${ }^{15}$ the $\mathrm{ADL},{ }^{15}$ and recovery ${ }^{15}$ ), but not an independent factor when strength or motor performance are included in the model. ${ }^{7,14,15}$ Only studies that have not measured strength or motor recovery have found sensation to be an independent factor in function and recovery. ${ }^{12,30}$ This finding suggests that the relationship between sensation and functional recovery exists because persons with sensory loss tend to have more severe weakness. Support for this hypothesis comes from 3 systematic reviews of the factors influencing recovery of ADL after stroke ${ }^{32-34}$ that have failed to identify sensory impairment as a significant independent negative prognostic indicator. However, one review ${ }^{33}$ suggested sensory loss is a negative factor for outcome.

Although proprioception and tactile sensation were related to function and recovery, a stronger correlation was seen when the 2 modalities were combined. This observation would suggest that both modalities should be measured to obtain a comprehensive indication of sensation in the research and clinical setting.

\section{Limitations of the Study}

Like all descriptive studies, the results of the present study are dependent on the sample recruited, the inclusion criteria, and the measurement tools used. Our inclusion criteria were designed to compose a typical rehabilitation population of persons with stroke. As only 9 patients who met the criteria declined to participate, we feel the sample is representative of our goal, but inevitably we recruited a minority of all persons with stroke and excluded the small number of persons with pure sensory stroke (less than $5 \%{ }^{35}$ ) and persons with very mild symptoms who were not admitted to the hospital. However, we feel the inclusion criteria were justified, as persons with a pure sensory stroke have a good prognosis and show a strong recovery, ${ }^{35}$ and they are rarely considered candidates for rehabilitation.

Despite the importance given to sensory impairments after stroke, there are relatively few measurement tools, and none have emerged as a gold-standard measure. We used some of the modalities from the RASP. ${ }^{20}$ In previous studies using the RASP, we have observed that sometimes the participants' level of attention and engagement in the testing process appear to influence the results. This influence was not found while testing other modalities such as weakness or neglect. To maintain the participants' attention throughout the testing procedure and thereby maximize the reliability of their performance, we limited the modalities tested to those we believed to be the most important, postural control, mobility, tactile sensation, and proprioception. This was a logical choice to maximize the quality of the data, but we may have lost useful information from other modalities such as vibration sense or stereognosis. The decisions made when using the RASP are relatively crude. They merely ask the participant whether he or she can feel something or not. Some other studies have used instrumented measurement tools to make more detailed tests of specific modalities, such as using an inclinometer to measure joint position sense ${ }^{5,9}$ or Semmes-Weinstein filaments to assess touch pressure. ${ }^{8}$ 
These measurement tools can be expected to be more sensitive and to identify a higher frequency of sensory impairment. However, they were unfeasible to use in a large cross-sectional survey. In the present study, we needed to test several modalities and several joints in both limbs. We included persons with a wide range of disabilities. We decided that using a measurement tool that reflected clinical practice and was suitable for use with a wide range of persons with stroke was the most appropriate choice for this study.

We also used 2 relatively crude measures of neglect, the Star cancellation and line bisection tests. ${ }^{18}$ Each of these tests has a defined score that indicates the presence of neglect. ${ }^{36}$ The use of a more sensitive test, such as the Test of Everyday Attention, ${ }^{37,38}$ may have given us a more detailed insight into the relationship between sensation and neglect/inattention, but the time required to complete it makes this test unfeasible for use in a large survey.

When comparing sensory loss at proximal and distal joints, we measured proprioception only in the upper limb. We did not search for a similar pattern in the lower limb or between different sensory modalities, or between different joints of the upper limb such as the shoulder. Further studies may address these issues.

\section{ACKNOWLEDGMENTS}

The authors acknowledge and thank the Stroke Association and Humphrey Booth Charities for funding the study. The authors have no competing interests.

\section{REFERENCES}

1. Carey LM. Somatosensory loss after stroke. Crit Rev Phys Rehabil Med. 1995;7:51-91.

2. Bobath B. Adult Hemiplegia: Evaluation and Treatment. 3rd ed. Oxford, UK: Butterworth-Heinnemann; 1990.

3. Partridge C, Cornall C, Lynch M, et al. Physical Therapies. In: Greenwood R, Barnes M, McMillan T, Ward C, eds. Neurological Rehabilitation. London: Mosby Hall; 1997.

4. De Haart M, Geurts A, Huidekoper SC, Fasotti L, van Limbeek J. Recovery of standing balance in post-acute stroke patients: A rehabilitation cohort study. Arch Phys Med Rehabil. 2004;85;886-895.

5. Lin S. Motor function and joint position sense in relation to gait performance in chronic stroke patients. Arch Phys Med Rehabil. 2005;86;197-203.

6. Lee MJ, Kilbreath SL, Refshauge KM. Movement detection at the ankle following stroke is poor. Aust J Physiother. 2005;51;19-24.

7. Feys H, de Weerdt W, Nuyens G, van de Winckel A, Selz B, Kiekens C. Predicting motor recovery of the upper limb after stroke rehabilitation: value of a clinical examination. Physiother Res Int. 2000;5:1-18.

8. Marigold D, Eng J, Tokuno CD, Donnelly CA. Contribution of muscle strength and integration of afferent input to postural instability in persons with stroke. Neurorehabil Neural Repair. 2004;18:222-229.
9. Niam S, Cheung W, Sullivan P, Kent S, Gu X. Balance and Physical Impairments after stroke. Arch Phys Med Rehabil. 1999;80: 1227-1233.

10. Leo KC, Soderberg GL. Relationship between perception of joint position sense and limb synergies in patients with hemiplegia. Phys Ther. 1981;61:1433-1437.

11. Han L, Law-Gibson D, Reding M. Key neurological impairments influence function-related group outcomes after stroke. Stroke. 2002;33:1920-1924.

12. Sommerfield DK, von Arbin MH. The impact of somatosensory function on activity performance and length of hospital stay in geriatric patients with stroke. Clin Rehabil. 2004;18:149-155.

13. Tyson S, Chillala J, Hanley M, Selley AB, Tallis RC. Distribution of weakness in the upper and lower limbs post-stroke Disabil Rehabil. 2006:28:715-719.

14. Tyson S, Chillala J, Hanley M, Selley AB, Tallis RC. Balance disability after stroke. Phys Ther. 2006:86;1;30-38.

15. Tyson S, Hanley M, Chillala J, Selley AB, Tallis RC. The relationship between balance disability and recovery after stroke: Predictive validity of the brunel balance assessment. Neurorehabil Neural Repair. In press.

16. Rankin J. Cerebral vascular accidents in patients over the age of 60. 2. Prognosis. Scott Med J. 1957;2:200-215.

17. Bamford J, Sandercock P, Dennis D, et al. A prospective study of acute cerebrovascular disease in the community; The Oxfordshire Community Stroke Project. 1: Methodology, demography, and incident cases of first-ever stroke. J Neurol Neurosurg Psychiatry. 1988;51:1373-1380.

18. Wilson B, Cockburn J, Halligan P. Development of a behavioral test of visuo-spatial neglect. Arch Phys Med Rehabil. 1987;68:98-102.

19. Collin C, Wade D. Assessing Motor Impairment after stroke; A pilot study. J Neurol Neurosurg Psychiatry. 1990;53:576-599.

20. Winward CE, Halligan PW, Wade DT. The Rivermead Assessment of Somatosensory Performance (RASP): standardisation and reliability data. Clin Rehabil. 2002;16:523-533.

21. Collin C, Wade DT, Davies S, Horne V. The Barthel ADL Index: A reliability study. Int Disabil Stud. 1988;10:61-63.

22. Collen F, Wade D, Robb F, Bradshaw CM. The Rivermead Mobility Index: A further development of the Rivermead Motor Assessment. Int Disabil Stud. 1991;13:50-54.

23. Smith DL, Akhtar AJ, Garraway WM. Proprioception and spatial neglect after stroke. Age Ageing. 1983;12:63-69.

24. Barnett HJM, Mohr JP, Stein BM, Yatsu FM, eds. Stroke: Pathophysiology, diagnosis and management. Vol 1. New York: Churchill Livingstone; 1986.

25. Botez MI. Parietal lobe syndromes In: Fredericks JAM, ed. Clinical Neuropsychology. Amsterdam: Elsevier Science; 1985.

26. Chester CS, McLaren CE. Somatosensory evoked response and recovery from stroke. Arch Phys Med Rehabil. 1989;70:520-525.

27. Reding M, Potes E. Rehabilitation outcome following initial unilateral hemispheric stroke: Life table analysis approach. Stroke. $1988 ; 19 ; 1354-1358$

28. Sanchez-Blanco I, Ochoa-Sangrador C, Lopez-Munain L, Izquierdo-Sanchez M, Fermoso-Garcia J. Predictive model of functional independence in stroke patients admitted to a rehabilitation programme. Clin Rehabil. 1999;13:464-475.

29. Kalra L. Crome P. The role of prognostic scores in targeting stroke rehabilitation in elderly patients. J Am Geriatr Soc. 1993;41:396-400.

30. Lai SM, Duncan PW, Keighley J. Prediction of functional outcome after stroke: comparison of the Orpington Prognostic Scale and the NIH Stroke Scale. Stroke. 1998;29:1838-1842.

31. Patel AT, Duncan PW, Lai SM, Studenski S. The relationship between impairments and functional outcomes poststroke. Arch Phys Med Rehabil. 2000;81:1357-1363. 
32. Kwakkel G. Wagenaar RC. Kollen BJ. Lankhorst GJ. Predicting disability in stroke-a critical review of the literature. Age Ageing. 1996;25:479-489.

33. Dombovy ML, Sandok BA, Basford JR. Rehabilitation for stroke: a review. Stroke. 1986;17:363-369.

34. Jongbloed L. Prediction of function after stroke: a critical review. Stroke. 1986;17:765-776.

35. Arboix A, Garcia-Pata C, Garcia-Eroles L, et al. Clinical study of 99 patients with pure sensory stroke. J Neurol. 2005;252;2;156-162.
36. Halligan P, Marshall JC, Wade DT. Visuospatial neglect: underlying factors and test sensitivity. Lancet. 1989;2:908-910.

37. Robertson IH, Ward T, Ridgeway V, Nimmo-Smith I. The structure of normal human attention: The Test of Everyday Attention. J Int Neuropsychol Soc. 1996;2:525-534.

38. Crawford JR, Sommerville J, Robertson IH. Assessing the reliability and abnormality of subtest differences on the Test of Everyday Attention. Br J Clin Psychol. 1997;36:609-617. 